Vol.02/ No. 02

Pages: 59-69

https://www.irojournals.com/jucct/

DOI: https://doi.org/10.36548/jucct.2020.2.001

\title{
Concept of Li-Fi on Smart Communication between Vehicles and Traffic Signals
}

\author{
Dr. S. R. Mugunthan, \\ Associate Professor, \\ Department of Computer Science and Engineering, \\ Sriindu college of Engineering and Technology, \\ Sheriguda, Hyderabad, India. \\ Email: srmugunth@gmail.com
}

\begin{abstract}
Communication is an essential thing for any work to be done on desired condition. There are several ways are there to communicate between a person to a machine. Switches are made as primary communication medium to do a specific task by a machine. The advancement of technologies introduced a remote switch for operation. The advancement is still continuing with voice recognition, hand gesture movement and mind reading models. Recently machine to machine communication was introduced to communicate their status between another machine or a system for implementing smart work on its own intelligence. The paper introduces a smart communication between vehicles to traffic signals and vehicle to vehicle using Li-Fi (Light Fidelity) technology for sharing necessary information to the nearby vehicle. The paper also describes the limitations and challenges in the Li-Fi technology for further communication improvement.
\end{abstract}

Keywords: Li-Fi, Bluetooth, Wi-Fi, Vehicle To Vehicle Communication, Traffic Signal Control.

\section{Introduction}

Communication between vehicles assist the driver to read about the traffic status and other several needful information. The communication can be done through several communication technology like Bluetooth, Wi-Fi and internet. All these communication technology has their own limitations and advantages. The Bluetooth communication has the ability to avoid interruption from the other kind of wireless devices but these communications are positive to information hacking. Also there are chances for disconnection in Bluetooth technology at certain climatic changes. This makes the communication system to be unstable in vehicle communication technology. The Wi-Fi technology is an alternate to Bluetooth device in terms of distance communication and increased efficiency. The Wi-Fi technology is not as like Bluetooth in terms of avoiding interruption from other communication signals. The speed performance on information sharing can also be affected when the connected devices are more in certain areas. Same as like of Bluetooth, WiFi information can also be easily hacked. 
Journal of Ubiquitous Computing and Communication Technologies (UCCT) (2020)

Vol.02/ No. 02

Pages: 59-69

https://www.irojournals.com/jucct/

DOI: https://doi.org/10.36548/jucct.2020.2.001

The data transmission between a vehicle to a nearby vehicle through internet can be efficient in terms of data security but the installation and communication cost will be very high than any other communication strategy. The internet communication need several blocks for data encryption, communication and decryption. A failure in the communication medium results in the whole system failure and makes the system in trouble mode. The proposed Li-Fi based communication is independent, encrypted data transmission, strengthen signal provider, faster data transmission and free from other communication interruption. The Li-Fi technology is reliable and can transmit several gigabytes of data at a stretch and secure than the Wi-Fi and Bluetooth technology. The interruption of radio frequencies from other medium is eliminated by transmitting the data through light waves.

The Li-Fi technology is simple and requires very low cost for design and performance. It doesn't require a complex circuit structure to produce radio frequencies production and antennas for transmission and receivers. The circuit which are needed for Li-Fi network is same amount of circuits required for a simple infrared transmission that is used in normal remote controls. By changing the intensity of the lights used for transmission, the Li-Fi can improve the amount of data transmission in the network. The improved efficiency and low latency makes the Li-Fi network is suitable for smart transport operations and vehicle communications. As the Li-Fi networks are free from radio waves it can be applied to several industrial and hospital applications for monitoring and operations. Figure 1 indicates the general structure of $\mathrm{Li}-\mathrm{Fi}$ technology.

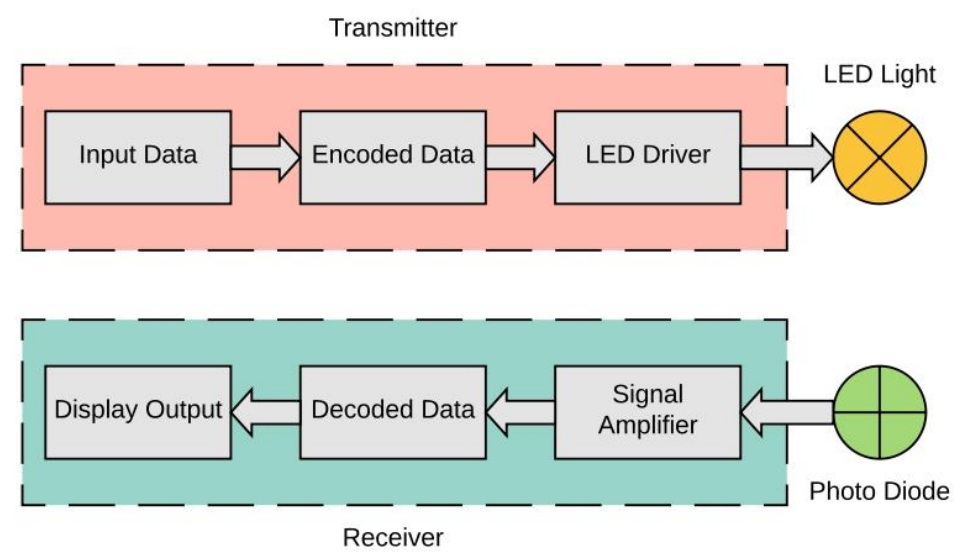

Figure 1. Structure of Li-Fi Technology

The general Li-Fi technology consists of a transmitter unit for encoding the given data into suitable binary data. The binary data with its corresponding PWM is generated by LED driver unit and forwards it to the LED light for blinking it with high intensity. The blinking speed in not able to visible for human eye as it 
Journal of Ubiquitous Computing and Communication Technologies (UCCT) (2020)

Vol.02/ No. 02

Pages: 59-69

https://www.irojournals.com/jucct/

DOI: https://doi.org/10.36548/jucct.2020.2.001

is working with very high frequency. The receiver unit consist of a photo diode to receive the light signal variations from the LED signal. The variations in the light makes the photo diode to generate various current changed output and the output current change is amplified by the signal amplifier for improving the quality of the signal. Then the signals are decoded to its original form for reading it to the display unit.

\section{Related Work}

This section explores various kind of vehicle communication system proposed already by using Bluetooth, Wi-Fi and Li-Fi technology. The intelligent system based on Bluetooth technology [1] was proposed to transmit the necessary information to the nearby roadside transmission station thorough Bluetooth medium and then the roadside station forwards the received to the intelligent base station for collecting the data through wired and Wi-Fi network. The system has a cooperative structure of several medium of communication for data transmission. The roadside stations which are connected to the base station receives the same information forwarded by the Bluetooth medium for managing the transport facility in the connected area. The vehicle to vehicle communication is carried by the help of a particular roadside station connected to several vehicles on that particular area. The system is developed communicate the nearby area with Bluetooth technology and faraway vehicles through internet connection. The advancement in 5G technology [2] mobile service is analyzed on its performance on various scenario and found the 5G technology has high speed in transmission rate of data with improved latency rate. A vehicle to vehicle communication was implemented using FPGA module and Wi-Fi network [3]. The implemented work is connected with the vehicle engine to monitor its speed and temperature using a specialized hardware connected to the engine. The hardware is connected with a microcontroller unit which was act as a brain for the system to transfer the signal received from engine to the other vehicle through Wi-Fi medium. The FPGA system improves the installation cost of the proposed system and the system also makes the system as a complicated one in computation programming. So that the system is also designed to adapt with Bluetooth technology for minimizing the cost needed for installation.

A virtual structure of wireless network [4] was designed with software defined networks, network virtualization function and deep packet inspection was analyzed on their own performance on network simulator tool. The software defined network comprises of three layers namely infrastructure layer for connecting the network device and transmitters, control layer with network service medium for transmitting the information pockets and an application layer. The network virtualization model is implemented here to virtualize the status of the network service. The deep pocket inspection model monitors the address of the data to be transferred by verifying its quality parameters. These kind of network model regulates the process with security in data transmission and confirmation of delivery. A dedicated short range communication between vehicles to vehicles [5] was proposed with a real time testing for an experimental study. The model is fixed with two numbers of 802.11 devices on two different vehicles. The experiment was done once by keeping the network device inside the vehicles and one time by keeping it over the roof of the vehicles. A 
Journal of Ubiquitous Computing and Communication Technologies (UCCT) (2020)

Vol.02/ No. 02

Pages: 59-69

https://www.irojournals.com/jucct/

DOI: https://doi.org/10.36548/jucct.2020.2.001

simple GPS device is fixed on both of the vehicles on their communication system. The system was tested by sending the GPS location and several files transferred through the USB device connected to the unit for performance evaluation. The experimental result indicates that the devices connected outside vehicle performs better than the devices connected inside in terms of connectivity.

An artificial intelligence based self-driving system [6] was developed by connecting several sensors for monitoring the surroundings of the vehicle and the status of the vehicle engine. The connected sensor information are summarized to a data collection unit for storage purpose. The stored data are preprocess and classified with an artificial intelligence processor for decision making. The decided information is passed to the electronic control unit for activating the vehicle performance by changing its speed, direction and performance. The vehicle control algorithm is also analyzed in the self-driving system. A wireless operated electric vehicle prototype [7] was made with Xbee, Bluetooth and Wi-FI based control unit. The performance of the vehicle can be controlled by a specially made android application on Bluetooth and WiFi protocols. The Xbee is controlled with a normal computer system. The Xbee protocol is simple and easy to implement but it can be useful for smaller distance. Bluetooth protocol can also be implemented for shorter distance communication as the Wi-Fi needs lot of control units. An IoT based smart home system [8] was proposed to connect appliances in a home to the concerned person available remotely. Along with the appliances certain parameters of the home environment are also measured by the help of sensors and forwarded to the beagle bone unit for transmitting the data through internet connection to the cloud system for storage and transmission. The data of certain emergency parameters are continuously monitored by the sensor but transfer the signal to the concern person only when there is an emergency situation. This minimizes the wastage of energy required for transmission and cloud storage.

A collision detection system [9] was designed to assist the vehicles on their driving by measuring the distance and speed of the nearby vehicle. The design is employed with an ultrasonic sensor for distance measurement and Li-Fi module for sending the speed information of the nearby vehicle to the following vehicle for collision avoidance. The proposed structure is controlled by a microcontroller unit connected with the sensor, Li-Fi transmitter and receiver for displaying the driving condition. A LCD display was fixed to indicate the distance between the vehicles and a servomotor controlled speedometer system was added to show the speed of the identified vehicle. The accuracy in measuring the distance between the vehicles was analyzed and regarding the speed the time needed to make the vehicle to zero was analyzed along with distance requirement. The survey on 5G network [10] was discussed to point out the advances and merits in the network system. The $5 \mathrm{G}$ networks are improved with latency, energy utilization and amount of data transmission per second are improved. The advancement in visible light communication [11] is listed out in the paper for understanding the working principle and applications of the Li-Fi technology. The Li-Fi technologies can overcome the disadvantages of the radio frequency systems due to its interference disturbance by the other source of radio waves. The system is widely used of vehicle to vehicle communication, underwater communication and certain hospital and industrial environment where the radio frequencies are avoided. 
Journal of Ubiquitous Computing and Communication Technologies (UCCT) (2020)

Vol.02/ No. 02

Pages: 59-69

https://www.irojournals.com/jucct/

DOI: https://doi.org/10.36548/jucct.2020.2.001

An automatic traffic signal controller [12] was developed to assist the emergency vehicles to cross the traffic signal areas easily. The system is developed by connecting Li-Fi transmitter and receiver on both side of normal vehicle and emergency vehicles. The emergency vehicle send a signal to the vehicle in front regarding the emergency situation and the vehicle standing in front transmit a signal to the Li-Fi receiver located on the traffic signal for identifying the situation to switch on the signal in to green for forwarding the emergency vehicle. The system is controlled by a pic microcontroller. A capsule network based traffic sign identification system [13] was made to recognize the road condition for smart driving. The system is fixed with a digital camera to capture the sign board signal and a preprocessing unit will be there for removing the noise and it enhances the images for classification algorithm. The capsule network based capsule network algorithm classifies the sign board with respect to the features extracted by the training data.

A theoretical analysis on Li-Fi technology [14] was made to find a hybrid solution for communication by adding Wi-Fi and LTE technology for different kind of location of transmitter and receiver. When the transmitter and receiver are too far in distance the communication is takes place using LTE network and when they are near to each other with in few meters the LTE network is change over to Wi-Fi network and the communication moves to Li-Fi only when the communication devices are very close to each other. Access point selection algorithm [15] was generated to balance the load distribution among the Li-Fi network and Wi-Fi network. A fuzzy logic algorithm is designed for selecting the access point with respect to the throughput and users count. The better throughput users are connected to the Wi-Fi network and the remaining users are connected to the Li-Fi networks for operations.

\section{Proposed Method}

The proposed smart communication between vehicles are achieved by sending signal from one vehicle to another vehicle through $\mathrm{Li}-\mathrm{Fi}$ medium. The $\mathrm{Li}-\mathrm{Fi}$ medium consists of transmitter unit for sharing information and receiver unit for receiving the signals. The transmitter is usually a LED light blinking with high frequency with various PWM depending upon the encoded information. Figure 2 explores that how the proposed system behaves on the real time application. All the vehicles involved in the study are fixed with two side Li-Fi modules, one fixed in front and another one fixed at the rear side of the vehicle. Both the modules are fixed with transmitter and receiver unit. Similarly both side of the vehicles are fixed with ultrasonic sensor for detecting the obstacles. The obstacles can be another vehicle coming closer to the primary vehicle. The ultrasonic sensor controls the Li-Fi unit and makes it 'ON' only when there is a vehicle closer to the primary vehicle. 
Journal of Ubiquitous Computing and Communication Technologies (UCCT) (2020)

Vol.02/ No. 02

Pages: 59-69

https://www.irojournals.com/jucct/

DOI: https://doi.org/10.36548/jucct.2020.2.001

\subsection{Vehicle to vehicle communication:}

The emergency vehicle Li-Fi units are comes to 'ON' position when they drive on siren mode. The emergency vehicles Li-Fi units continuously send a signal to the front transmitter like 'emergency vehicle is following give way'. If the roads are fixed with Li-Fi modules it will be able to send the lane number on where the emergency vehicle is moving. The vehicle which receives the signal from the emergency vehicle can move from the lane where the emergency vehicle is moving. This system makes a better visibility to the rear side view of a vehicle and it avoids the confusion in changing the lane when there is an emergency vehicle. This improves the safety in driving.

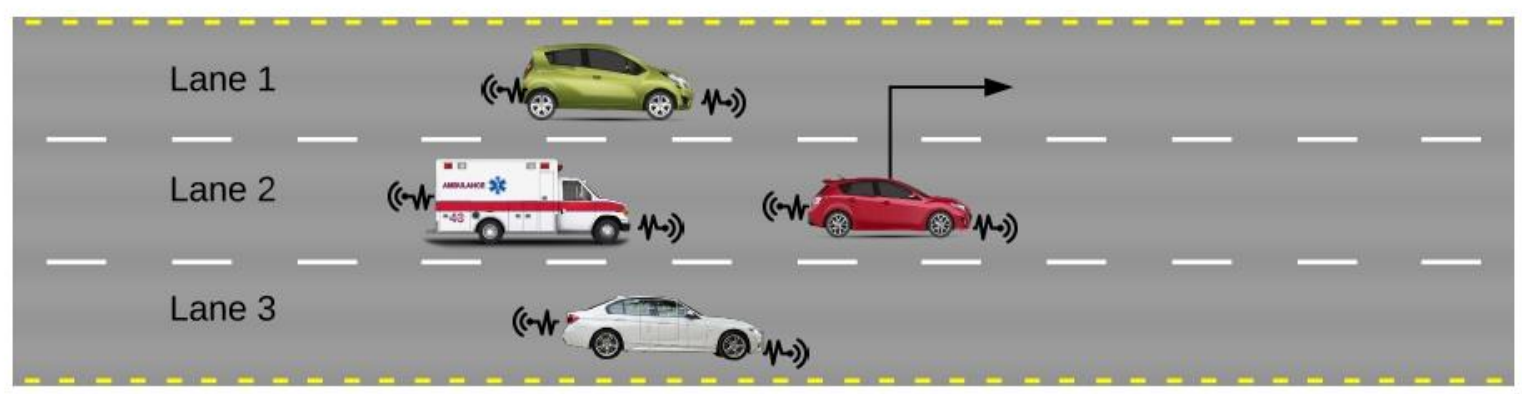

Figure 2. Structure of vehicle to vehicle communication.

\subsection{Emergency vehicle to traffic signal communication: (Direct method)}

During vehicle to traffic signal communication the transmitter on the emergency vehicle sends a signal about its status to the nearby receivers on vehicles, roads and traffic signal post. Figure 3 explores how the $\mathrm{Li}-\mathrm{Fi}$ modules are fixed over the road and connected to the traffic signal for power transmission. The Li-Fi modules on the road are always on when the traffic signals are in working condition. The modules can be molded with a heavy material casing for its safety on vehicle movement over the module. The modules on the road can be kept 16 feet once for making a better communication to the vehicles standing in the signal. In these kind of communication the message from an emergency vehicle is transmitted directly to the traffic signal for making it to green. Similarly the vehicles standing in front of the emergency vehicle are also gets a message on their display like 'emergency vehicle on lane 3 go forward' and at the same time the vehicle standing on the other lane get a message like 'stop emergency vehicle moving on lane 3' to avoid congestion on the traffic signal area. 
Vol.02/ No. 02

Pages: 59-69

https://www.irojournals.com/jucct/

DOI: https://doi.org/10.36548/jucct.2020.2.001

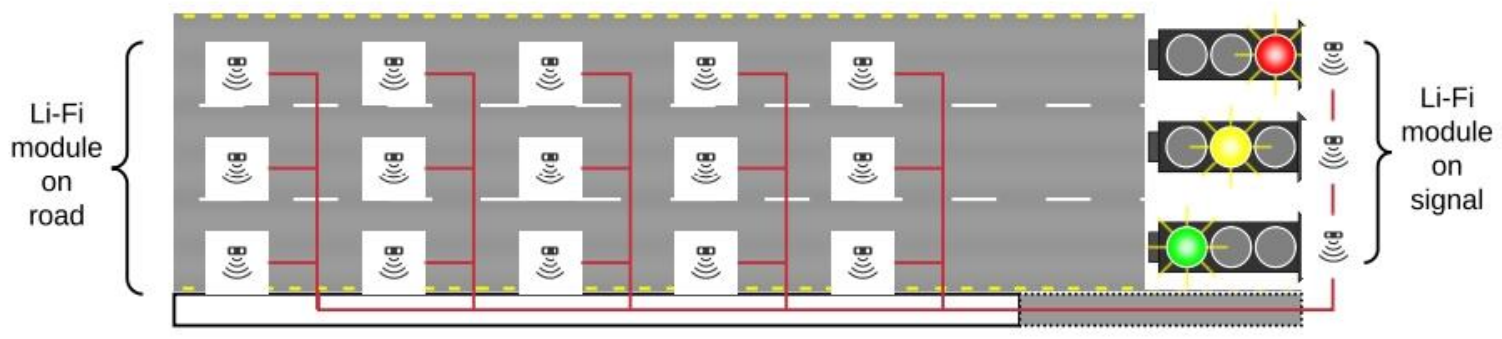

Figure 3. Wiring Connection of Li-Fi Modules on Road

\subsection{Emergency vehicle to traffic signal communication: (Indirect method)}

The communication between the emergency vehicles to the traffic signal can also be communicated directly by passing the signal to its front vehicle to front vehicle for reaching the traffic signal post receiver. This saves the cost requirement on fixing the Li-Fi module over the road. The vehicle receives a signal from an emergency vehicle will display the status of the following to its display and at the same time it forwards the message to the neighbor vehicle stating that 'emergency vehicle on your lane move forward'. The vehicle waiting in the first row forward the signal to the traffic post for changing the signal in to green on such particular lane alone. The remaining lane vehicle signals are in stop mode. Figure 4 explicates the model of these communication process.

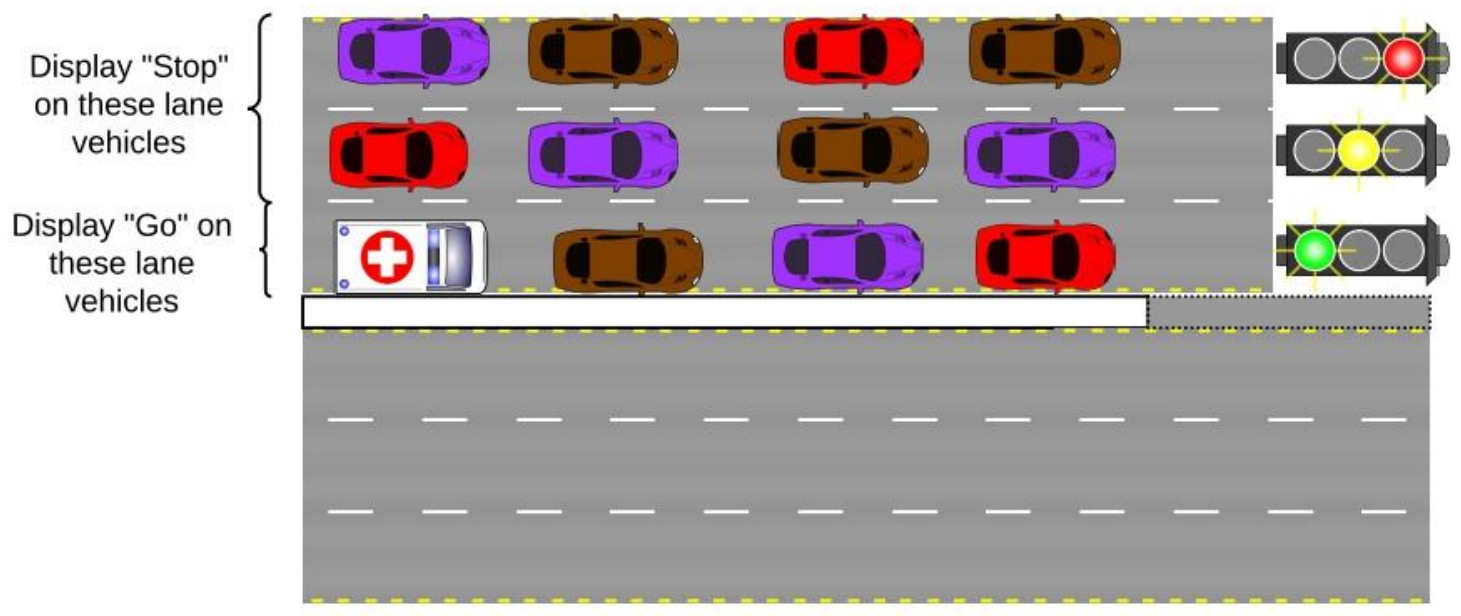

Figure 4. Structure Of Emergency Vehicle To Traffic Signal Communication Through Nearby Vehicle Communication. 
Journal of Ubiquitous Computing and Communication Technologies (UCCT) (2020)

Vol.02/ No. 02

Pages: 59-69

https://www.irojournals.com/jucct/

DOI: https://doi.org/10.36548/jucct.2020.2.001

\subsection{Module setup:}

The proposed method is verified with an arduino simulator tool. The Li-Fi modules are made by the help of LED lights and photo diode. Two numbers of arduino boards were used in the setup. One acts an emergency vehicle Li-Fi module and the other one acts as a nearby vehicle module or traffic signal module. The LED lights are acting as a transmitter on the modules at the same time the photo diodes are acting as the receiver on the modules. The change in PWM of the LED light connected with the emergency vehicle describes a specific message to the nearby vehicle. The received messages are displayed on the $16 \times 2$ LCD display fixed over the receiver side Li-Fi module. The LED lights connected separately to the breadboard acts a traffic signal setup, makes the signal 'ON' depending upon the received message. The arduino board has an inbuilt slot for PWM changes which can be used for changing the frequency of operation of the LED.

\section{Experimental Results}

Figure 5 indicates the simulator result of the proposed Li-Fi based smart communication system. A LCD display is fixed with the arduino board for displaying the received message from the transmitter module. The receiver arduino is fixed with a Li-Fi module for receiving the signals from the transmitter module. Depending upon the frequency of the light blinked on the transmitter module, the result are indicated on the vehicle side display as well as the traffic signal light. The receiver module on each lane is designed to display different messages. The result image indicates the display of a vehicle standing on the same lane of an emergency vehicle. So that the traffic signal LED and "Go Forward" messages were displayed at the same time. Similarly, the information about the emergency vehicle location on road is also displayed on the LCD display first row.

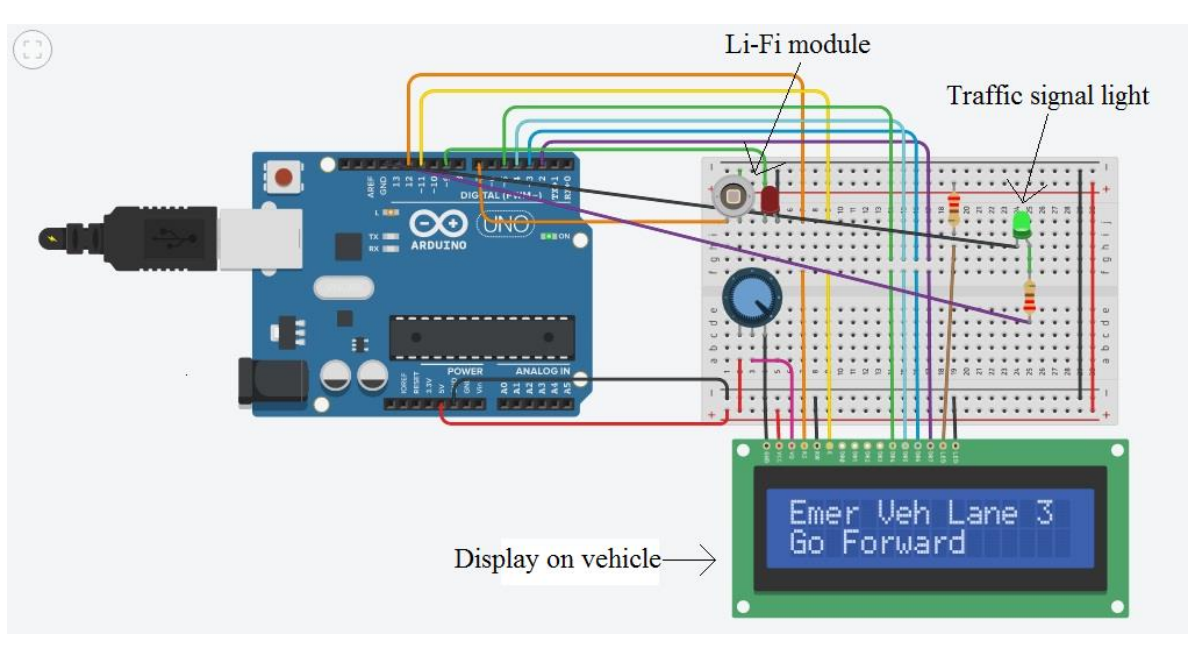

Figure 5. Proposed Smart Communication Receiver Module During Result Display 
Journal of Ubiquitous Computing and Communication Technologies (UCCT) (2020)

Vol.02/ No. 02

Pages: 59-69

https://www.irojournals.com/jucct/

DOI: https://doi.org/10.36548/jucct.2020.2.001

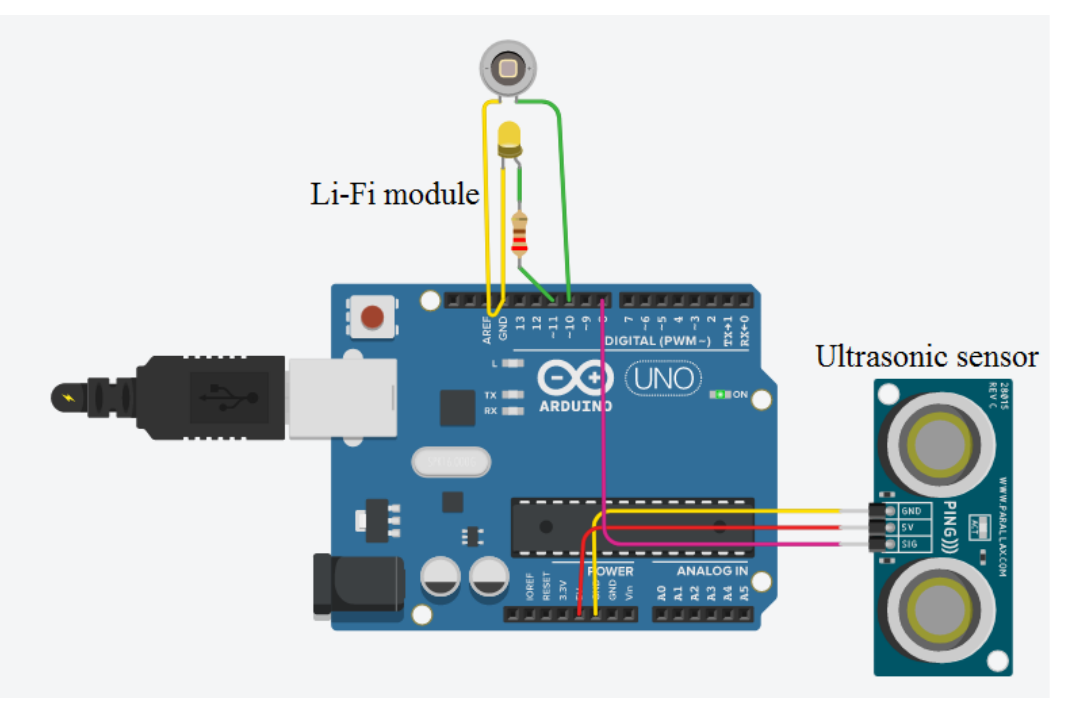

Figure 6. Proposed Smart Communication Transmitter Module During Transmission.

Figure 6 shows the transmitter portion of the Li-Fi module. The transmission part moves to 'ON' position only when a vehicle is coming closer to the emergency vehicle. An ultrasnonic sensor is fixed for such application to the transmitter side arduino board. The ultrasonic sensor continuously ssense the neraby surroundings for obstacle identification and it send a signal to the microcontroller when there is an obstacle near to the vechile. The obstacle can be a vehicle moving closer in front of the emergency vehicle. After realising that the microcontroller allows the Li-Fi transmitter to send the necessary signal to the receiver module. The transmitter LED is blinking with different PWM depending upon the information to be transmitted. The transmission information is changed with respect to the road side Li-Fi modules information received by the emergency vehicle module regarding the road and lane number to be displayed.

\section{Conclusion}

The proposed Li-Fi technology based smart communication for vehicle to vehicle and vehicle to traffic signal is simulated with an arduino simulator tool and the results are obtained. The Li-Fi technology of transmission is simple and free to radio signals interruption. But the transmitting signals are affected to the sunlight and other lights on the road. High frequecy of LED light is needed to overcome such issues. Comparitively the range of transmission of the Li-Fi technology is also lesser than the existing Bluetooth and Wi-Fi technology. Also the transmitter and receiver has to be kept almost to the same position for the transmitter and receiver module for efficient data transmission. The successful communcation of Li-Fi module can be affected by just blocking the transmitter LED by a material. Unless in Bluetooth and Wi-Fi modules the signals are allowed to cross over the obstacles. The proposed methodolgy can be implemented in real time operation by covering up the limitations of the Li-Fi technology using a perfect design to coverup the transmitter and receiver module from atmosphere damages and blockages. 
Journal of Ubiquitous Computing and Communication Technologies (UCCT) (2020)

Vol.02/ No. 02

Pages: 59-69

https://www.irojournals.com/jucct/

DOI: https://doi.org/10.36548/jucct.2020.2.001

\section{References}

[1] Thomas, Kévin, Hacène Fouchal, Stephane Cormier, and Francis Rousseaux. "Intelligent Transport System Based on Bluetooth." In International Workshop on Communication Technologies for Vehicles, pp. 50-59. Springer, Cham, 2019.

[2] Chen, Joy Iong Zong. "5G technology and advancements in connected living-comprehensive survey." Journal of Electronics 1, no. 02 (2019): 71-79.

[3] Shrestha, Pratik, Binayak Subedi, Megan Girard, Chirag Parikh, and Nabeeh Kandalaft. "Wireless Communication Between FPGA and Microcontroller." In 2020 10th Annual Computing and Communication Workshop and Conference (CCWC), pp. 0402-0405. IEEE, 2020.

[4] Raj, Jennifer S., and S. Smys. "Virtual structure for sustainable wireless networks in cloud services and enterprise information system." Journal of ISMAC 1, no. 03 (2019): 188-205.

[5] Hoque, Mohammad A., Jackeline Rios-Torres, Ramin Arvin, Asad Khattak, and Salman Ahmed. "The extent of reliability for vehicle-to-vehicle communication in safety critical applications: an experimental study." Journal of Intelligent Transportation Systems 24, no. 3 (2020): 264-278.

[6] Manoharan, Samuel. "An improved safety algorithm for artificial intelligence enabled processors in self driving cars." Journal of Artificial Intelligence 1, no. 02 (2019): 95-104.

[7] Champaty, Biswajeet, Suraj Kumar Nayak, Goutam Thakur, Biswajit Mohapatra, D. N. Tibarewala, and Kunal Pal. "Development of Bluetooth, Xbee, and Wi-Fi-Based Wireless Control Systems for Controlling Electric-Powered Robotic Vehicle Wheelchair Prototype." In Robotic Systems: Concepts, Methodologies, Tools, and Applications, pp. 1048-1079. IGI Global, 2020.

[8] Duraipandian, M., and Mr R. Vinothkanna. "Cloud based Internet of Things for smart connected objects." Journal of ISMAC 1, no. 02 (2019): 111-119.

[9] Krishnan, Prabu. "Design of Collision Detection System for Smart Car Using Li-Fi and Ultrasonic Sensor." IEEE Transactions on Vehicular Technology 67, no. 12 (2018): 1142011426.

[10] Valanarasu, Mr R., and A. Christy. "Comprehensive survey of wireless cognitive and 5G networks." Journal of Ubiquitous Computing and Communication Technologies (UCCT) (2019): 23-32.

[11] Khan, Latif Ullah. "Visible light communication: Applications, architecture, standardization and research challenges." Digital Communications and Networks 3, no. 2 (2017): 78-88.

[12] Shanmughasundaram, R., S. Prasanna Vadanan, and Vivek Dharmarajan. "Li-Fi Based Automatic Traffic Signal Control for Emergency Vehicles." In 2018 Second International Conference on Advances in Electronics, Computers and Communications (ICAECC), pp. 1-5. IEEE, 2018.

[13] Koresh, Mr H. James Deva, and J. Deva. "Computer vision based traffic sign sensing for smart transport." Journal of Innovative Image Processing (JIIP) 1, no. 01 (2019): 11-19. 
Journal of Ubiquitous Computing and Communication Technologies (UCCT) (2020)

Vol.02/ No. 02

Pages: 59-69

https://www.irojournals.com/jucct/

DOI: https://doi.org/10.36548/jucct.2020.2.001

[14] Lambrechts, Wynand, and Saurabh Sinha. "A Theoretical Analysis of Li-Fi: A Last Mile Solution." In Last Mile Internet Access for Emerging Economies, pp. 109-142. Springer, Cham, 2019.

[15] Islam, Rashed, and M. Rubaiyat Hossain Mondal. "Hybrid DCO-OFDM, ACO-OFDM and PAM-DMT for dimmable LiFi." Optik 180 (2019): 939-952.

[16] Wu, Xiping, Majid Safari, and Harald Haas. "Access point selection for hybrid Li-Fi and WiFi networks." IEEE Transactions on Communications 65, no. 12 (2017): 5375-5385.

\section{Authors Biography}

Dr. S. R. Mugunthan, is presently working as an Associate Professor, in the Department of Computer Science and Engineering, in Sriindu college of Engineering and Technology, Sheriguda, Hyderabad, India. His area of research includes ubiquitous networks, Big Data Analytics, Industrial System and Collective Intelligence on all fields of Computing and Communication Technologies. 